María Auxiliadora Linares*

\title{
La indagación fenomenológica: herramienta para el abordaje del aprendizaje del diseño arquitectónico ${ }^{1}$
}

\author{
The phenomenological inquiry: tool for approaching \\ architectural design learning
}

Cómo citar:

Linares, M. (2017). La indagación fenomenológica: herramienta para el abordaje del aprendizaje del diseño arquitectónico. Designia, 5(1), 69-83.

${ }^{1}$ Este artículo forma parte de las reflexiones surgidas dentro de la investigación "Competencias Investigativas: aproximación teorética a sus significaciones para la integración de saberes curriculares en

el Taller de Diseño Arquitectónico", desarrollada en el contexto docencia investigativa del Departamento de Teoría y Práctica de la Arquitectura y el Diseño, Programa de Arquitectura, Facultad de Arquitectura y Diseño de la Universidad del Zulia, Venezuela.

*Arquitecta de la Universidad del Zulia, Venezuela. 1988. Magíster Scientiarum en Ciencias de la Educación. Universidad Dr. Rafael Belloso Chacín, Venezuela. 2004. Experta en Procesos E-Learning. Fundación para la Actualización Tecnológica de Latinoamérica, World Wide Web. 2013. Finalizando Tesis

Doctoral. Doctorado en Educación. Universidad Pedagógica Experimental Libertador, Venezuela (2015-2017). Profesora Asistente del Programa de Arquitectura de la Facultad de Arquitectura y Diseño de la Universidad del Zulia. (2008-actualidad). Directora del Instituto Universitario de Tecnología Juan Pablo Pérez Alfonzo, Extensión Maracaibo, Venezuela. (2008-actualidad). fadmariauxlinares@gmail.com
Palabras clave:

Arquitectura, fenomenología, aprendizaje, investigación, pedagogía del diseño.

Key words:

Architecture, phenomenology, learning, research, pedagogy of design.

Recibido: 02/02/2017

Aceptado: 04/03/201

\section{Resumen:}

El presente artículo de reflexión surge a partir de indagaciones sistematizadas en la praxis académica y la inquietud de orientar a los estudiantes del taller de Diseño Arquitectónico de la Universidad de Zulia, Venezuela, hacia estrategias investigativas transformadoras en su proceso de aprendizaje. 
Se parte de la diversidad de variables que devienen de la propia complejidad de la disciplina, en tanto ciencia que posibilita el accionar humano en espacios habitables, centro neurálgico del hacer arquitectónico. Este entramado complejo encuentra en la Fenomenología una herramienta valiosa para el estudiante en su abordaje indagatorio durante el proceso de aprendizaje y en su futura vida profesional. A partir de un análisis hermenéutico de carácter documental sobre los postulados de autores como Bachelard, Husserl y Van Manen, se deriva el aporte del método fenomenológico al aprendizaje de la Arquitectura. A su vez, se apalanca el estudio de experiencias vividas que sirven de información experiencial, las cuales se recogieron de la voz de habitantes a través de instrumentos fenomenológicos como entrevistas, registros anecdóticos y observaciones de campo. Los profesores del Taller de Diseño Arquitectónico pueden encontrar en la indagación y la descripción fenomenológica una ruta vivencial para el abordaje académico del diseño del espacio arquitectónico.

\section{Abstract:}

This paper is derived from systematized inquiries related to the academic praxis and the concern for guiding the students of the Architectural Design Workshop of the University of Zulia, Venezuela, to innovative research strategies in their learning process. The starting point are the variables associated to the complexity of the discipline as a science that enables human actions in living spaces. Phenomenology represents a valuable tool for such a complexity as it may support students in questioning during their learning process as well as in their professional work. From a hermeneutical analysis on the postulates of authors such as Bachelard, Husserl and Van Manen, the contribution of the phenomenological method to architectural learning is identified. Phenomenological instruments such as interviews, recording of particular cases and field observations allowed to know experiences from inhabitants of some places. Such experiences served as experiential information to be studied. Professors of Architectural Design Workshop may find in inquiring and the phenomenological description a direction for the academic approach of the architectural space design.

El espacio captado por la imaginación no puede seguir siendo el espacio indiferente entregado a la medida y a la reflexión del geómetra. Es vivido. Y es vivido, no en su positividad, sino con todas las parcialidades de la imaginación

(Bachelard, 1957, p. 22) 


\section{INTRODUCCIÓN}

La temática de este artículo de reflexión emerge de inquietudes compartidas sobre el hacer académico de los estudiantes de Arquitectura y de la indagación respecto a cómo estos pueden abordar procesos investigativos más allá de lo observable a simple vista, los cuales les permitirían, a posteriori, comprender el significado otorgado por las personas a los espacios donde habitan y el modo en que aspirarían habitarlos. Esto con el propósito de brindar nuevos escenarios de aprendizaje y enseñanza de la disciplina que propulsen el abordaje de la investigación no solo desde el hecho arquitectónico sino también, y principalmente, desde las vivencias de quienes lo habitan.

Desde esa perspectiva, primero se indaga de manera rasante, dada la profundidad de sus fundamentos, en la Fenomenología como método de investigación de la tradición cualitativa que enfatiza en el estudio de las experiencias vividas a partir de lo expresado por las propias personas, en este caso los habitantes del lugar y sus significaciones. Para ello, se consideran los aportes de notables fenomenólogos, tales como Husserl, Bachelard y Van Manen, así como de Alexander y Einseman dentro de la propia Arquitectura.

Posteriormente, se presentan los aportes de la Investigación Pedagógica Fenomenológica Hermenéutica (IPFH) propuesta por Van Manen (2003), que en su desarrollo ofrece una serie de actividades de investigación que en virtud de su origen pedagógico bien pudieran adaptarse o contextualizarse a la investigación en Arquitectura. Esta base serviría de referente para los estudiantes hacia la creación de una propia metodología de trabajo indagatorio.

Por último, se ofrecen conclusiones que pueden orientar investigaciones académicas relacionadas con el tema, como también servir de sustentación para los profesores en su hacer cotidiano, en su permanente interés por abrir oportunidades de aprendizaje a sus estudiantes de Arquitectura.

\section{LA INVESTIGACIÓN Y SU ROL EN EL HACER ACADÉMICO}

La evolución del ser humano está signada por la investigación, toda vez que el origen de esta se fundamenta en la innata curiosidad surgida de la necesidad de saber y comprender. Su utilidad puede únicamente y de modo general evocar a las mentes de la ciencia, no obstante, se evidencia hasta en lo más mínimo de nuestra cotidianidad, siempre orientada por el interés, más aún en áreas en que la práctica o la aplicación de hallazgos constituyen el centro de atención. 
Vista como proceso, la investigación se inicia en la indagación, discurre en la sistematicidad de esta y se afianza en la autocrítica consciente que motiva a ir cada vez más allá de lo hallado. Asumida como proceso sistemático y autocrítico (Stenhouse, 1993), la investigación en el ámbito universitario generalmente se visualiza como una función profesoral y no estudiantil, al menos no en los primeros años de estudios, pues se piensa que el estudiante solo debe ocuparse de estudiar. Sin embargo nos preguntamos: ¿qué es estudiar si no es investigar?

$\mathrm{Al}$ respecto, en términos del aprendizaje, la relevancia de la investigación para el estudiante universitario puede ser focalizada de dos modos. Primero, desde el objeto de estudio, propicia la proximidad gradual y directa hacia los contenidos y los modos de aplicarlos al procurar las fuentes originarias del conocimiento, al igual que la comprensión de este como resultado. Segundo, desde el estudiante en sí, la investigación ofrece herramientas motivadoras del aprendizaje autónomo, del ordenamiento mental de ideas y procesos. A su vez, potencia el pensamiento holístico, crítico y complejo, toda vez que ayuda a crear estructuras asociadas con subprocesos mentales, tales como exploración, análisis, síntesis, comprensión e interpretación, entre otras competencias que permiten entender realidades y necesidades en un contexto académico, social o profesional en aras de crear, descubrir y proponer soluciones a problemas complejos (Parra, 2004).

Con la visión de la investigación como estrategia didáctica integradora, Parra (2004) enfatiza en lo que denomina investigación formativa, la cual "trasciende su función puramente docente para convertirse en una estrategia pedagógica de mayor alcance: lograr una formación universitaria basada en el trabajo científico de los estudiantes" (p. 73). Al presentarse dos modos de concebir y visualizar la investigación -estrategia pedagógica y proceso sistemático autocrítico- se infiere que debe utilizarse a diario en las aulas y propiciar su desarrollo como herramienta de trabajo estudiantil y hábito para la formación profesional.

En el caso que nos ocupa, el aprendizaje de la Arquitectura, la investigación debe ser empleada, además, como herramienta para el desarrollo de la creatividad y la innovación, en consonancia con las competencias propuestas por el Proyecto Tuning América Latina (2011-2013) para los egresados al finalizar su formación, entre las cuales se destaca la "capacidad de aplicar los métodos de investigación proyectual para resolver con creatividad las demandas del hábitat humano, en diferentes escalas y complejidades" (Vélez, 2013, p. 34). Ello implica, en primer lugar, conocer y manejar métodos investigativos que permitan al estudiante construir un conocimiento que ha de proseguir a posteriori en la vida profesional. 


\section{LA CURIOSIDAD Y EL SABER: COMPONENTES PARA LA VIDA UNIVERSITARIA EN ARQUITECTURA}

El conocimiento de los seres humanos tiene sus raíces profundas en la curiosidad, en esa intrínseca necesidad de saber qué hay detrás de los eventos, de las situaciones, de los fenómenos que nos rodean. ¿Cómo?, ¿por qué? y ¿para qué? son interrogantes que desde niños y por siempre mantenemos en nuestras mentes, los cuales nos conducen en mayor o menor grado, según sea nuestra capacidad de curiosear y nuestro interés hacia lo curioso, por un camino hacia el encuentro de respuestas a esas inquietudes surgidas en nuestro interior.

Del grado de importancia que para nosotros tenga el asunto causante de nuestro interés, de nuestra capacidad de indagación, de la repercusión del evento en nuestra vida y en la de otros, así como de los hallazgos de la búsqueda, dependerá la trascendencia de ese proceso indagatorio. Su desenvolvimiento como proceso conducirá hacia distintos rumbos. A detenernos, porque nos satisface la respuesta obtenida; a tomar de nuevo los avíos de la indagación y continuar nuestro andar al haber encontrado otros matices, rutas o maneras de abordaje; o bien, del camino anterior, puede surgir un nuevo objeto de nuestra curiosidad que nos impulsa de manera inquietante a indagar en él.

Visualizamos lo anterior a la manera de un tornado curioso que se apalanca en la motivación por saber. Se genera un conflicto cognitivo capaz de impelernos al pensamiento creativo, el cual plantea y produce variadas e innovadoras soluciones fundadas en nuestros conocimientos previos y en aquellos que, a lo largo de la búsqueda, vamos adquiriendo. En el recorrido indagatorio, con ojo avizor, conseguimos respuestas que devienen en comprensiones, explicaciones o propuestas, según las características del evento motivador de la indagación.

La investigación, para quien se forma como arquitecto, representa el puente entre la curiosidad y el saber. Se trata de un proceso surgido de la motivación, de un impulso por comprender algo, por dar respuesta a una necesidad. "Un acto de investigación es una acción para impulsar una indagación" (Stenhouse, 1984, p. 88). En el contexto académico de la carrera de Arquitectura, investigar debe animar, 
desencadenar naturalmente en los estudiantes esa indagación, dadas sus cualidades de conducir hacia la búsqueda de respuestas y la comprensión de fenómenos, de aportar soluciones a necesidades en la disciplina y en el ámbito personal del estudiante, en su modo de apropiarse del conocimiento.

Como hacer y profesión, el aprendizaje de la Arquitectura comporta la exploración de soluciones convenientes al hábitat humano con respeto al medio ambiente, ya sea natural o construido, para lo cual considera múltiples variables, término utilizado en la disciplina para referir los aspectos que intervienen, y por ende, deben tenerse presentes en la consecución del hecho arquitectónico ${ }^{1}$.

La Arquitectura debe ser abordada desde varios planos y apelar a otras áreas para comprenderse de manera integral. "Debe recurrir para explicarse a conceptos y procesos de otras especialidades -sociales, antropológicas, urbanas, tecnológicas, estéticas, históricas, etc.- que aparentemente siempre la determinan" (Suárez, 2007, p. 48A). De lo anterior se visualiza una suerte de tejido multicolor, de carácter complejo por naturaleza, que constituye el campo del saber arquitectónico. Hacia este se dirigen todas esas semillas de curiosidad surgidas en el ser de los estudiantes, las cuales los propulsan a querer buscar, a indagar en las razones y en los porqués de la disciplina.

Esa naturaleza compleja de la Arquitectura da cuenta de la belleza palpable a través de su condición artística; del espacio, presente en su carácter funcional; de la técnica, producto de su origen científico; de lo contextual, en virtud de estar localizada en un lugar determinado; y lo más importante, acude a sentimientos enraizados en su esencia humanística, entendida dentro del campo antropológico y sociológico, ya que en ella confluyen conceptos psicológicos, socioantropológicos y espirituales del ser humano. Así, es una mezcla variopinta de variables y relaciones que resultan en un hecho de características únicas.

Se infiere entonces que el estudiante debe enfrentar tres realidades que le serán

${ }^{2}$ Destacamos que este término se aplica con fines de determinar una dinámica cualitativa más que cuantificable. propias como futuro Arquitecto. Estas son la social, la cutural y la espacial. A estas se auna su propia vivencia como ser pensante y cargado de experiencias. Por lo tanto, el profesor tiene la tarea de servir de guía en el andar entre esas realidades e incentivar la resolución de problemas inherentes al hecho arquitectónico derivado de los contextos en los cuales este se inserta. 
El desarrollo del futuro arquitecto depende de tres grupos de capacidades: de sentir, relacionar y comprender las realidades que le rodean; de obtener información, procesarla y compartirla; de visualizar una solución, luego plasmarla, comunicar la idea de manera gráfica y materializarla en un hecho arquitectónico. A grandes rasgos, esto muestra el proceso no solo de diseño arquitectónico sino también de aprendizaje que debe gestarse para la formación.

Tal multiplicidad de capacidades interrelacionadas valida esta idea: "proyectar es un ordenamiento intelectual, una clarificación de conexiones, una definición de dependencias, una ordenación de pesos, y presupone una especial capacidad en la cabeza del proyectista para ver y fijar analogías, conexiones, campos relacionales" (Aicher, 2001, p. 181).

Para Aicher (2001), el arquitecto no puede considerarse un científico, pues no piensa en términos de lógica y conclusiones. El arquitecto relaciona, por lo tanto, no piensa de modo lineal sino que teje redes, dispone y ordena, enlaza sistemas y subsistemas, genera organizaciones espaciales, valora la optimización del espacio que le es propio. En este sentido, sostiene una relación dialógica entre las realidades de su contexto y sus propias realidades (sensorial, perceptiva, reflexiva y creadora), en una suerte de sinergia permanente, en la cual la investigación no puede quedar de lado. En este punto, la Fenomenología, como modo de emprender el camino investigativo, entra en juego para aportar luces al estudiante al proceder en la indagación para el aprendizaje de la Arquitectura.

\section{APORTES DEL CAMPO DE LA FENOMENOLOGÍA AL APRENDIZAJE DE LA ARQUITECTURA}

En 1957 Gastón Bachelard publicó La poétique de l'espace (La poética del espacio), obra que aborda de manera magistral la relación entre Fenomenología y Arquitectura al entender la casa como el espacio vivencial por excelencia. El autor presenta una metodología para conocer y comprender los fenómenos que ocurren en tal lugar a partir del complejo y cotidiano hecho de habitarlo, y cómo esto refleja el alma humana. Para tal fin, se apoya en postulados comparativos entre la psiquis y un edificio, propuestos por el célebre psicólogo Carl Jung.

Analicemos esta reflexión: "Porque la casa es nuestro rincón del mundo. Es -se ha dicho con frecuencia- nuestro primer universo. Es realmente un cosmos. Un cosmos en toda la acepción del término. Vista íntimamente, la vivienda más humilde ¿no es la más bella?" (Bachelard, 1957, p. 28). Es clara la alusión a las significaciones de la casa y cuanto esta involucra para quien la habita: su intimidad, su cosmos, su 
ideal de belleza más allá de la estética visual. Puede hablarse de una estética del alma. De lo anterior, nos preguntamos: ¿quién mejor para referirnos un lugar que quien allí reside? Sus respuestas, más aún si son espontáneas, arrojan pistas para imaginar, dibujar, diseñar y crear, pues remiten a la cultura de esa persona, a sus necesidades, valores y aspiraciones, a sus maneras de visualizar el mundo, y en consecuencia, de vivirlo.

Esa visión intimista, humana, fenomenológica para indagar, es posible enlazarla con la de Christopher Alexander, quien en su obra de 1981, El modo intemporal de construir, para intentar definir ese "modo" expresa (p. 22):

\section{Se trata de un instinto humano primordial, tan intrínseco como el deseo de tener hijos. Es sencillamente el deseo de hacer parte de la naturaleza, de completar un mundo que ya está formado (...) con algo hecho por nosotros y que también forme parte de la naturaleza y de nuestro entorno inmediato.}

Desde la visión de este arquitecto, para adentrarnos en la comprensión de un edificio, de un lugar, no debemos hacerlo visualizando el hecho arquitectónico como un objeto, sino como resultado de lo humano y de lo vivido.

Orientada a indagar en lo vivido, la Fenomenología se relaciona con la intuición y la percepción de los eventos, desde una perspectiva esencial de lo vivido (Álvarez, 2013). Impulsada por Edmund Husserl (1859-1938), parte de la descripción de las entidades presentes en la intuición intelectual y logra captar la esencia de estas, aquella que trasciende a la consciencia.

En relación con la Arquitectura, el campo que nos ocupa, asumida como una trama multidimensional, por ende compleja y compuesta por eventos, es posible abarcarla desde la óptica pedagógica de la Fenomenología, la cual sirve de método de indagación de lo interno en las situaciones vividas. "La arquitectura puede ser entendida como fenómeno, entonces podría ser materia de estudio de la Fenomenología" (Ocampo, 2014, p. 99). Esto a partir de la "relación entre el sujeto que habita y lo que percibe. Dicho planteamiento ubica al individuo en el espacio en actitud perceptiva y dispuesto a la inferencia" (Ocampo, p. 99). 
El complejo entramado de variables que conforman el espacio arquitectónico, mediado en su comprensión por la Fenomenología, puede hablarnos de una significativa habitabilidad para quien lo vive. Ocampo (2014) alude a Maurice Merleau-Ponty (1908-1961), filósofo fenomenológico francés, quien en 1948 presentó una serie de conferencias sobre percepción en las cuales planteó la indagación del espacio y del lugar como exploración del mundo percibido.

A partir de los planteamientos fenomenológicos y de su relación con lo vivido, surge la Investigación Fenomenológica Pedagógica Hermenéutica (IFPH), expuesta por Max Van Manen en su libro publicado en 2003, Investigación Educativa y Experiencia de Vida, cuyo centro neurálgico es la descripción de la cualidad vivida, que bien pudiera estar referida a la intrigante "cualidad sin nombre" (Alexander, 1981, p. 11). Dicha cualidad emerge de todo cuanto nos rodea, que a pesar de su sencillez y evidencia, en la mayoría de ocasiones es difícil de captar. ¿O quizá, de buscar? En esta búsqueda, la IFPH tiene su punto de origen en la descripción fenomenológica de corte pedagógico, orientada por la intencionalidad de esclarecer adecuadamente algún aspecto de la realidad, aquello que atrapa nuestro interés, pues al no ser comprendido, comienza a retumbar en nuestro modo de entender la vida y nos impele a indagar.

Esa experiencia vivida en el espacio habitable interesa a quien trabaja en Arquitectura, ya sea estudiante, profesor o profesional, en tanto a partir de costumbres, estilos, vivencias, necesidades, expectativas, modos de ser, de vivir y de sentir, se desprende la esencia de la disciplina como ciencia, arte y rama del saber, cuyo accionar se centra en los espacios habitables y los seres que los habitan. Al respecto, cuando queremos hacer Arquitectura "hay que decir, pues, cómo habitamos nuestro espacio vital de acuerdo con todas las dialécticas de la vida, cómo nos enraizamos, de día en día, en un 'rincón del mundo'"' (Bachelard, 1957, p. 28). Esto hace alusión a la necesidad de adentrarnos más allá del objeto-espacio, sea este una casa, escuela, hospital, parque, oficina o tantos otros inherentes al hacer del arquitecto, para conocer a quien los vive, constituye y transforma en espacios vividos, y desde allí emprender el acto de diseñar.

Para la Fenomenología, el ambiente concreto es el lugar y cuanto sucede en este representa un evento digno de observar. En consecuencia, el lugar consiste en cosas concretas que tienen la sustancia material, la forma, la textura y el color. Estos elementos, en su conjunto, de acuerdo con quien los observe, perciba o viva, se funden para formar el carácter del ambiente. Esta cualidad sin nombre, al modo de Alexander (1981), permite a ciertos espacios, con funciones similares e incluso 
idénticas, encarnar propiedades distintas, en consonancia con las condiciones culturales y ambientales del lugar donde existen. En este sentido, la Fenomenología aporta a la Arquitectura una postura ontológica, epistemológica, axiológica y metodológica; otro enfoque al problema del espacio arquitectónico.

\section{La Fenomenología trata del estudio de las esencias; la Arquitectura posee la capacidad de hacer resurgir las esencias. Relacionando forma, espacio y luz, la Arquitectura eleva la experiencia de la vida cotidiana a través de los múltiples fenómenos que emergen de los entornos, programas y edificios concretos. Por un lado, existe una idea/fuerza que impulsa la Arquitectura; por otro, la estructura, el material, el espacio, el color, la luz y las sombras intervienen en su gestación (Holl, 1997, p. 11).}

Esas esencias devienen en los espacios, como resultado de la interacción entre estos y los seres que los habitan. Sentimientos, percepciones, emociones, sensaciones, no solo desde la óptica de quien vive el espacio, sino también de quien lo ha proyectado o construido. Al intentar entender las significaciones que un recinto patrimonial tiene para una determinada ciudad, no basta con indagar en los libros de historia, sino también y primeramente en las personas: proyectista, constructor, usuarios, residentes, trabajadores, turistas. Asimismo, como arquitectos podemos indagar si en el espacio conviven plantas y animales, y analizar sus propias vivencias a partir de la observación. Es posible entonces examinar la luz natural que incide en un lugar para el crecimiento de una flor o un árbol, cómo utiliza un gato un sitio para dormir la siesta, o cómo las personas se sientan frente a la fachada de un edificio y se dejan bañar por sus memorias.

Todas esas indagaciones arrojan pistas sobre la esencia del lugar y su significación, tanto para sus habitantes como para la ciudad, las cuales son de gran utilidad al analizar, entender o intervenir un espacio patrimonial. Así, a partir del carácter reflexivo en la exploración de la experiencia vivida, es posible abordar desde la IFPH el aprendizaje y la subsecuente práctica de la Arquitectura, como profesión y área del saber, de acuerdo con los siguientes planos de perspectiva ontoepistémica que son descritos de modo general.

Plano Ontológico: al considerar la coexistencia en la Arquitectura de múltiples realidades emergentes y socialmente construidas, inmersas en un entramado complejo de interrelaciones formativas de índole académico, en las cuales confluyen además otras de carácter personal que estructuran el ser y el saber de cada actor 
educativo. Se busca entender esa praxis vivencial que da significado a los objetos y a los hechos, y cómo estos dan significado a las personas. Se pretende "descubrir lo que subyace en el centro ontológico de nuestro ser" (Van Manen, 2003, p. 31), es decir, qué piensan, sienten, opinan y vivencian los seres en relación con el evento o fenómeno en estudio, pues es "la complejidad y el misterio de la vida lo que exigen reflexión” (Van Manen, p. 35).

Plano Axiológico: fundamentado en el reconocimiento de valores, creencias, opiniones y experiencias vividas por las personas. Considera los saberes de estas, así como sus propias vivencias en constante construcción y deconstrucción, a través de un proceso dialógico intersubjetivo desde el cual emergen las esencias. En este sentido, la Fenomenología se orienta al conocimiento del cosmos humano tal como lo encontramos en el universo: heterogéneo, diverso, cambiante, en su forma natural o con el rigor que proviene de la moral y la ética. Ese cosmos tiene su centro en el lugar donde habita, su casa, su sitio de trabajo, de estudio, de entretenimiento. En los espacios habitables el sentido del ser humano se afianza en tanto echa raíces y se apropia de ellos, para llenarlos de sus propias significaciones (Van Manen, 2003).

Plano Epistemológico: considera el conocimiento no como producto del intelecto humano sino resultante de una comprensión de los hechos, es decir, como un proceso continuo de construcción y desconstrucción, un "geistig, una cuestión de profundidad del alma, del espíritu, la expresión personificada del saber y el ser" (Bollnow en Van Manen, 2003, p. 32). Por lo tanto, debe asumirse una perspectiva que primero visualiza y luego indaga en la persona del ser humano, en el sentido pleno de la palabra. Entiende que de la observación e investigación de la práctica de vida en los espacios arquitectónicos habitables sobreviene la teoría, el proyecto y el edificio como producto de un proceso de interpretación hermenéutica y reflexión fenomenológica en torno a las esencias desveladas para comprender el fenómeno en estudio.

Plano Metodológico: la Fenomenología tiene su punto de origen en la descripción fenomenológica, orientada por la intencionalidad de esclarecer adecuadamente algún aspecto de la vida, aquello que atrapa nuestro interés. Esa descripción se recoge de las experiencias vividas y, a su vez, permite validarlas (Van Manen, 2003). El recorrido de un lugar a otro a través de un tercero que los une supone un cúmulo de experiencias 
vividas: observaciones, percepciones, emociones que pueden recogerse por medio de conversaciones, entrevistas, diarios anecdóticos, dibujos, fotografías y hasta el arte callejero. A través de una interpretación hermenéutica reflexiva es posible extraer las esencias significativas del lugar como insumo para el hacer arquitectónico.

Las seis actividades de investigación planteadas por la IFHP (Van Manen, 2003) sirven de guía a quienes buscan el apoyo de la Fenomenología como modo de indagación en la Arquitectura. En especial para los estudiantes, en su proceso de aprendizaje, representan un método para desarrollar su propia aproximación al espacio habitable y a las significaciones de este para quienes lo habitan. De manera general, tales actividades, llevadas al contexto de la Arquitectura, son:

1.- Centrarse en la experiencia vivida. A partir de la observación del fenómeno en estudio, en este caso el espacio arquitectónico y su significado para las personas dentro del contexto en el cual está inmerso el hecho arquitectónico.

2.- Investigar la experiencia como es vivida. Mediante entrevistas en profundidad realizadas a los informantes, en este caso los habitantes del lugar, se indagan sus vivencias, anécdotas, pareceres y saberes. También es válido solicitarles fotografías, escritos, dibujos, en fin, recurrir a elementos que según la naturaleza del tema arquitectónico, sean un aporte para comprender el evento en estudio.

3.- Reflexionar sobre los temas esenciales. El análisis hermenéutico de las transcripciones de las entrevistas y los demás materiales reunidos, en conjunto con la consecuente meditación y reflexión fenomenológica, permiten esclarecer el panorama investigativo. Para ello, el profesor de Arquitectura debe ser un guía y orientador del estudiante durante su proceso reflexivo.

4.- El arte de escribir y reescribir. Mediante el lenguaje, la experiencia vivida se convierte en un discurso reflexivo que da cuenta del fenómeno y lo visualiza tal y como es. En Arquitectura, a partir de los hallazgos, ese discurso comienza a tomar forma en ideas comunicativas de diseño que se sintetizan en bocetos, dibujos y notas que estructuran poco a poco las primeras ideas. 
5.- Mantener una relación firme y orientada. Esto es necesario en la reflexión fenomenológica para fijar una posición de interés científico, con ánimo y sentido humano que permita profundizar en la búsqueda de la verdad de la experiencia vivida por los informantes, en la esencia de sus sentires y pensamientos. Quienes habitan el lugar son o serán parte de esos espacios, por tanto, son o serán los encargados de validar el accionar del arquitecto.

6.- Explicar el contexto investigativo. Para tal fin es valioso un texto o gráfico explicativo con organización argumentada, cuya estructura dialéctica sea consecuencia directa de lo extraído de las partes. Así, a partir de estas se construye el todo explicativo con una estructura global, textual y gráfica, una especie de Gestalt reflexiva que es el resultado de desvelar la esencia del proyecto de diseño arquitectónico y sus elementos constituyentes.

La Arquitectura puede así trascender la forma. El edificio como hecho construido pasa a condensar una compleja relación con los sujetos que lo habitan.

\begin{abstract}
La arquitectura superará el postulado racionalista de la forma geométrica, admitiendo la forma y el movimiento, no ya como principios, sino como fenómenos: la espiral que envuelve, los planos que giran. El punto de llegada del análisis fenomenológico demostrará la necesaria variabilidad de la forma por la continua variación del exterior, por el devenir mismo de la realidad y por la continua variación del interior, por el devenir de los puntos de anclaje del sujeto (Álvarez, 2013, p. 832).
\end{abstract}

\section{CONCLUSIONES}

El mundo de lo sensible es el mundo primigenio de la Arquitectura, percibida como ciencia social cuyo centro de estudio es el espacio y el ser humano que lo habita, o en otras palabras, la idea del espacio habitable y habitado por el ser humano. Por ende, son las personas, los habitantes del lugar, quienes pueden expresar con idoneidad sus propias vivencias ante la indagación arquitectónica. En ese sentido, la Fenomenología aporta un componente de indagación vivencial a la práctica del diseño arquitectónico, toda vez que a través de la percepción sensorial del mundo, el arquitecto adquiere información del espacio que le rodea.

El contacto perceptivo propulsado a profundidad por la corriente fenomenológica ofrece un sinnúmero de herramientas que van de la mano con nuestro hacer como arquitectos, profesores o estudiantes de Arquitectura: entrevistas, observaciones que trascienden la vista e involucran el resto de los sentidos, materiales como fotografías, 
dibujos, escritos y videos, elaborados por el propio estudiante-arquitecto o por los habitantes del lugar, y la posibilidad de volver a estos para confirmar y validar la información. Tales recursos permiten la indagación, inferencia y posterior reflexión hermenéutica arquitectónica, tanto para el aprendizaje de la disciplina como para su hacer profesional.

La Arquitectura, en tanto ciencia humana, encuentra en la Fenomenología un apoyo para el hacer del diseño arquitectónico. Gracias a esta es posible recurrir al habitante, al usuario, al ser humano y sus vivencias como hacedor de espacios habitables. Esto es de gran valía para la indagación de aspectos que las ciencias exactas no pueden ofrecer, pues permite reconocer el significado del lugar para quien lo habita, desde la óptica y el relato de quienes le dan o dieron vida.

La experiencia vivida interesa al hacedor de Arquitectura, ya que le facilita un acercamiento, de modo intimista y enriquecedor, al centro mismo de la necesidad de habitar. De este modo, se acrecienta su sensibilidad ante los requerimientos, las aspiraciones y los intereses de las personas, con lo cual podrá expresar en sus propuestas de solución una óptica más apegada a la realidad que dio origen a la necesidad. Aun así, siempre pensará que habrá una solución mejor, pues la subjetividad humana posee límites insospechados que otorgan a la Arquitectura su carácter permanente en tanto sea significativa para quien la vive.

En síntesis, la Fenomenología es una herramienta de sumo valor para la pedagogía del diseño arquitectónico. Queda en manos de los profesores de los talleres de diseño arquitectónico apelar a ella como instrumento metodológico para posibilitar en los estudiantes el acercamiento al espacio desde adentro, desde la experiencia vivida, con el propósito de desvelar su esencia a través de la descripción fenomenológica de los espacios habitables. 


\section{REFERENCIAS BIBLIOGRÁFICAS}

Aicher, O. (2001). Analógico y digital. Barcelona: Gustavo Gili.

Alexander, C. (1981). El modo intemporal de construir. Barcelona: Gustavo Gili.

Álvarez, L. (2013). Arquitectura y fenomenología. Sobre la arquitectónica de la "indeterminación" en el espacio. Eikasia, 47(47), 815-836. Recuperado de http://www.revistadefilosofia.org/47-47.pdf

Bachelard, G. (1957). La poética del espacio [Ernestina de Champourcin, trad.]. Recuperado de https://monoskop.org/images/1/16/Bachelard_Gaston_La_poetica_del_espacio.pdf

Holl, S. (1997). Entrelazamientos. Barcelona: Gustavo Gili.

Ocampo, J. (2014). La lectura de textos clásicos fenomenológicos en la formación de arquitectos. Enunciación, 19(1), 93-106. Recuperado de http://revistas.udistrital.edu.co/ojs/index.php/enunc

Parra, C. (2004). Apuntes sobre investigación formativa. Educación y Educadores, 7, 70-73. Recuperado de http://www.redalyc.org/articulo.oa?id=83400707

Stenhouse, L. (1993). La investigación como base de la enseñanza. Madrid: Morata.

Suárez, J. (2007). La multidimensionalidad permanente de la Arquitectura desde el enfoque de la metafilosofía de la ciencia. Portafolio, 7, 47-50A. Recuperado de http://www.produccioncientificaluz.org/ index.php/portafolio/article/view/12795/12782

Van Manen, M. (2003). Investigación educativa y experiencia vivida. Recuperado de https://es.scribd.com/ mobile/doc/172841140/Libro-Investigaion-educativa-y-experiencia-de-vida-Van-Manen

Vélez, S. (2013). Educación Superior en América Latina: reflexiones y perspectivas en Arquitectura. Proyecto Tuning América Latina Recuperado de http:/tuningacademy.org/wp-content/uploads/2014/02/ RefArchitecture_LA_SP.pdf 